\title{
Wahrscheinlichkeitstheoretische Modelle in der Schadenversicherung
}

\section{Doctoral Thesis}

Author(s):

Kupper, Josef

Publication date:

1962

Permanent link:

https://doi.org/10.3929/ethz-a-000087590

Rights / license:

In Copyright - Non-Commercial Use Permitted 
Prom. Nr. 3178

\title{
Wahrscheinlichkeitstheoretische Modelle in der Schadenversicherung
}

\author{
Von der \\ EIDGENŐSSISCHEN TECHNISCHEN \\ HOCHSCHULE IN ZURICH \\ zur Erlangung \\ der Würde eines Doktors der \\ Mathematik \\ genehmigte \\ PROMOTIONSARBEIT
}

Vorgelegt von

JOSEF KUPPER

dipl. Math. ETH

von Luzern und Buttisholz (LU)

Referent: Herr Prof. Dr. W. Saxer

Korreferent: Herr Prof. Dr. H. Wyss

Gedruckt 1962 in Würzburg bei Konrad Triltsch 


\section{Wahrscheinlichkeitstheoretische Modelle in der Schadenversicherung}

Die Schadenversicherung kann von zwei verschiedenen Warten aus betrachtet werden. Der eine, ältere Gesichtspunkt gründet auf dem Risiko des einzelnen Versicherten und ist unter dem Namen individuelle Risikotheorie bekannt. Seit $F$. Lundberg [50] ${ }^{*}$ ) hat die kollektive Betrachtungsweise immer mehr Einfluß auf die Versicherungstheorie gewonnen, was aus den zahlreichen Arbeiten über diesen Gegenstand (Arfwedson, Cramér, Philipson, Segerdahl; Ammeter, Saxen, Campagne u.a.) hervorgeht. Diese befaßt sich nicht mehr mit dem Risiko eines einzelnen Individuums, sondern untersucht das Verhalten einer versicherten Gruppe als Ganzes. Die individuelle Theorie geht beispielsweise davon aus, ein wahrscheinlichkeitstheoretisches Modell herzuleiten, das darüber Auskunft erteilt, mit welcher Wahrscheinlichkeit ein Versicherter in einer gewissen Zeitspanne $\mathbf{r}$ Schäden erleide; die kollektive Schau hingegen bemüht sich vorerst darum, dasselbe für einen ganzen Versicherungsbestand zu erfahren, ungeachtet dessen, was mit den individuellen Versicherungen in dieser Zeit geschieht. Da die beiden Anschauungen in ihrem methodischen Aufbau weitreichende Analogien aufweisen, soll in dieser Arbeit nicht starr an einem Gesichtspunkt festgehalten werden, sondern je nach den besonderen Absichten sei der eine oder andere mehr in den Vordergrund gerückt.

Die Schadenversicherung - wir denken, wenn von Schadenversicherung die Rede ist, hauptsächlich an den wichtigen Zweig der Unfallversicherung - basiert auf zwei stochastischen Größen, der Schadenzahl und der Schadenhöhe. Hierin tritt bereits ein fundamentaler Unterschied zur Lebensversicherung zutage, wo letztere in den weitaus meisten Fällen eine zum voraus festgelegte, feste Zahl darstellt. Die interessantere der beiden Variablen ist die Schadenzahl; ihre Verteilung wird im umfangreichen Kapitel I diskutiert. Wir beschränken uns dabei zum vornherein auf die Verteilung in einer bestimmten Zeitspanne (Zeiteinheit), gehen also nicht auf Fragen der zweidimensionalen Behandlung (= zwei Zeitperioden, siehe z.B. [9], [13]) ein.

Ausgangspunkt der Betrachtung bildet das klassische Modell des reinen Zufalls $(\S 1)$, das unter sehr einschränkenden Bedingungen über den zu Grunde liegenden Prozeß erhalten wird. Insbesondere ist, worauf bereits Greenwood und Yule in ihrer bahn. brechenden Arbeit [38] hingewiesen haben, Homogenität des Bestandes und Unab. hängigkeit der Ereignisse Voraussetzung.

Die Aufhebung des ersten Grundsatzes kommt als Gegenstand von § 2 zur Sprache. Seit Greenwood/Yule [38] und Newbold [53,54] wird für die dabei auftretende Struk. turfunktion (Verteilungsfunktion der verschiedenen Schadenanfälligkeit) fast ausschließlich eine Gamma-Verteilung verwendet. Greenwoods eigene Begründung [36] „Our only justification of the particular choice of $f(\lambda)$ was that it ranged from $\lambda=0$ and led to a statistically useful form" sowie die Erklärung von Bates und Neyman [13] ,,is justified both by its flexibility as an interpolation formula and by the tradition established by Greenwood, Yule and Newbold" sind nicht so überzeugend, um nicht auch Raum für Versuche mit anderen Strukturfunktionen durchzuführen. Als wichtiges Hilfsmittel erweisen sich hierbei die konfluenten hypergeometrischen Funktionen. Schließlich wird auch die Möglichkeit der Binomialverteilung als Grund. verteilung samt den daraus sich ergebenden Erweiterungen einer kurzen Analyse unterzogen.

*) Zahlen in [ ] beziehen sich auf das Literaturverzeichnis am Schluß der Arbeit. 
Das Gebot der Unabhängigkeit der Ereignisse kann nach zwei Richtungen hin gelockert werden. Das Kollektivschadenmodell (§3) nimmt an, daß ein Ereignis Ursache mehrerer Schäden sein könne. Es werden zwei allgemeine Darstellungen für das Kumulrisiko und verschiedene instruktive Beispiele gegeben. $\S 4$ ist dem Ansteckungsmodell gewidmet, dem die Voraussetzung zu Grunde liegt, daß das Eintreffen (bzw. Nichteintreffen) eines Schadenereignisses den weiteren Schadenverlauf maßgeblich beeinflußt. Wir gehen von einem ganz allgemeinen Schema aus, das jedoch in Spezialfällen bekannte Modelle (Eggenberger/Pblya [26, 27], Rutherford [63]) enthält.

In einem letzten Paragraphen sind schließlich einige gemischte Modelle (Utberlagerung der zuvor besprochenen Eigenschaften) diskutiert.

Kapitel II befaßt sich mit der Verteilung der Schadenhöhe. Verschiedene Anregungen zur Behandlung dieser Frage werden unterbreitet und an Hand zahlreicher Beispiele beleuchtet.

Endlich hat Kapitel III die Zusammenfassung von Schadenzahl und Schadenhöhe zum Totalschaden als Thema. Dabei wird an der Unabhängigkeit der beiden stochastischen Grundvariablen festgehalten. Während in $\$ 1$ die übliche Methode zur Darstellung gelangt, erläutern die folgenden Paragraphen einen etwas anderen Aspekt des Problems. An die Stelle der Schadenzahl tritt die Schadenhäufigkeit als Grundvariable. Da diese ihrer Definition nach besser durch eine stetige Verteilung wiedergegeben wird (Normalverteilung!), erhebt sich die Frage einer Anpassung des Totalschadenmodells. Zwei Lösungswege werden vorgeschlagen, die Einführung der stetigen Faltung (\$3) und die Zuhilfenahme der Produktverteilung (\$4).

Zwecks Klarlegung der Begriffe und Bezeichnungsweisen sowie im Interesse einer flüssigen Arbeitsweise sind in einem Anhang die wichtigsten, überall in die Rechnung eingehenden Funktionen und Verteilungen mit ihren Eigenschaften im Sinne einer protokollarischen Übersicht aufgezeichnet. 